\title{
Quasistatic Compression of a Compact Torus
}

\author{
A. W. Molvik, J. L. Eddleman, J. H. Hammer, C. W. Hartman, and H. S. McLean \\ Lawrence Livermore National Laboratory, Livermore, California 94550
}

(Received 1 October 1990)

\begin{abstract}
We have demonstrated the formation of stable, symmetric, compact-torus (CT) plasma rings and the subsequent stable, twofold radial compression in coaxial conical electrodes with the ring accelerator experiment. The $\mathbf{C T}$ is compressed by $\mathbf{J} \times \mathbf{B}$ forces from a capacitor bank discharging across the conical electrodes. During compression, the force of the $\mathbf{B}_{\theta}$ acceleration field balances the force of the CT poloidal field against the cones, in good agreement with a 2D MHD code. Power amplification factors of $\sim 100$ may be possible with an opening switch based on this technique.
\end{abstract}

PACS numbers: $52.55 . \mathrm{Hc}, 52.75 . \mathrm{Di}, 52.75 . \mathrm{Kq}$

We report the first quasistatic compression of a compact torus (CT), using the $\mathbf{B}_{\theta}$ toroidal field of a coaxialelectrode, rail-gun-type accelerator to compress the CT through a coaxial cone into a straight coaxial geometry where the CT is accelerated. These experiments were performed in RACE, the ring accelerator experiment. ${ }^{1}$ This is a significant step toward inductive energy storage for acceleration and achieving the higher power densities and magnetic-field values required for many applications of accelerated CTs, ${ }^{2}$ including an inertial-confinementfusion driver. ${ }^{3}$ Parameters achieved in RACE are already suitable for two applications: (1) The 2-T magnetic fields, $0.16-\mathrm{m}$ diameters, and the $10-100-\mu \mathrm{g}$ ring masses are appropriate for fueling and current-drive experiments in tokamaks; ${ }^{2-7}$ and (2) the large disparity in $\mathbf{B}_{\theta}$-field time scales between the compression phase $(10-30 \mu \mathrm{s})$ and the acceleration phase ( $2300 \mathrm{~ns})$ suggests that an "opening switch" 8 or power amplifying device with amplification factors $\simeq 100$ could be possible.

Quasistatic compression of a CT, frequently called a spheromak, ${ }^{9,10}$ has been demonstrated elsewhere in a different geometry. (We refer to the RACE plasma configuration as a CT or spheromak, even though it has a conductor along the axis, because the essential physics is the same.) Inductively formed CTs in the S-1 experiment ${ }^{9}$ were compressed by a factor of 1.3-1.6, using a pair of $100-\mu \mathrm{s}$-rise-time coils to compress the CT deeper into the cylindrical "vee" formed by a pair of funnelshaped flux conservers. The experiments on RACE achieve a larger compression factor of 2 in the major and minor radii, by forcing the CT through the annulus between two coaxial cones, as shown in Fig. 1. Image currents in the metal walls constrain the CT fields to remain localized radially and axially in the interelectrode gap and help maintain stability during compression. On RACE, the compression ends with the CT entering a straight coaxial acceleration stage where it is free of axial constraints and can be accelerated to serve a variety of purposes.

The concept of a CT accelerator, as embodied in RACE, is first to form the CT with a magnetized coaxial plasma gun, ${ }^{11-13}$ and then to accelerate it by the $\mathbf{J} \times \mathbf{B}$ force with separately powered coaxial electrodes. ${ }^{2}$ The plasma-gun discharge between coaxial electrodes produces a toroidal $\mathbf{B}_{\theta}$ field, coupled with a solenoidal magnetic field that links the electrodes. Under sufficient pressure from the $\mathbf{B}_{\theta}$ field, the solenoidal field balloons out, forming the CT poloidal field when reconnection occurs. ${ }^{12,14}$ Subsequent relaxation yields a long-lived CT configuration approaching the minimum-energy Taylor state. ${ }^{15}$ For these experiments, the gas injected into the gun has been hydrogen, argon, or neon. Following formation, a second capacitor bank is discharged through an insulating feed that does not link the gun and therefore produces a $\mathbf{B}_{\theta}$ field only. The pressure of the $\mathbf{B}_{\theta}$ field can compress the CT in conical electrodes, as we describe, and/or accelerate the CT over a distance of



FIG. 1. The ring-accelerator-experiment apparatus, consisting of a magnetized plasma gun, a precompression cone, and 1.7-m-long coaxial accelerator. Magnet probes are located at $0.12 \mathrm{~m}$ and at $0.43 \mathrm{~m}$. Other probes, not shown, are used for acceleration data in Fig. 4. 
many times its own length to large directed energies, as has been demonstrated previously. ${ }^{1}$ A combination of compression, acceleration, and a final focusing stage is predicted to reach the very high power densities (e.g., the $>10^{15} \mathrm{~W} / \mathrm{cm}^{2}$ at $10-\mathrm{MJ}$ energy) required for an inertial-fusion driver. ${ }^{3}$

RACE is now configured with a precompressor cone following the plasma gun, which quasistatically compresses the torus to a diameter of $0.16 \mathrm{~m}$ at the entrance to a straight accelerator $1.7 \mathrm{~m}$ long, shown in Fig. 1, for the experiments described in this paper. Reconnection of magnetic-field lines into a CT configuration after exiting the gun is now aided by having the inner electrode step inward while the diameter of the gun's outer electrode remains constant. Forming a CT with the same diameter as that of the gun was tried, in part, due to the recent success on the CTX facility in making smaller CTs this way. ${ }^{10,16}$ An earlier configuration of RACE consisted of a 6-m-long, 0.5- (0.2-) m outer (inner) diameter straight coaxial accelerator, expanded from a $0.32-\mathrm{m}$ gun, with a focusing cone at the end. ${ }^{1}$ This configuration demonstrated acceleration to $1 \%$ of the speed of light, and focusing to $0.16-0.25 \mathrm{~m}$ from an initial $0.5-\mathrm{m}$ diameter as inertial forces carried the CT into the cone.

There are several advantages to employing ring formation in compression cones as the first stage of a CT accelerator. (1) The compression cone serves as a flux conserver in which slow gun operation builds up a CT over many characteristic Alfvén times, allowing a lower voltage and current, higher-inductance, and hence lowerpower capacitor bank to drive the plasma gun. ${ }^{10,17}$ (2) The CT is stable within the cone and becomes azimuthally symmetric after the gun turns off. (3) When the gun is turned off, the CT magnetic field decays linearly to zero over a relatively long period $(60 \mu \mathrm{s})$, making the firing time of the accelerator capacitor bank less critical than when the accelerator must be synchronized within a few $\mu$ s with a fast gun that produces a CT velocity ${ }^{1}$ of typically $\sim 2 \times 10^{7} \mathrm{~cm} / \mathrm{s}$. (4) A time of order $10 \mu \mathrm{s}$ is required for the accelerator bank to compress the CT through the cone to the straight section where it can be accelerated. During this time, energy is being inductively stored behind the CT, ready to be quickly released when the torus reaches the straight section. This enables a slower, lower-power capacitor band to also be used for acceleration.

Stable CTs are usually formed, as shown in Fig. 2(a) by data from the azimuthal array of four magnetic loop probes, spaced every $90^{\circ}$ at the beginning of the precompressor. The $d \mathbf{B} / d t$ signal of the probes is digitized, then numerically integrated. Small asymmetries are seen at the beginning of the formation, but these damp out after the gun turns off at 30-35 $\mu$ s. Even when the CT shows a large asymmetry early in time [Fig. 2(b)], the asymmetry dies out within $\sim 30 \mu \mathrm{s}$, leaving an azimuthally symmetric and quiescent $\mathrm{CT}$ by the time the accelerator is fired at $60 \mu \mathrm{s}$. These data demon-

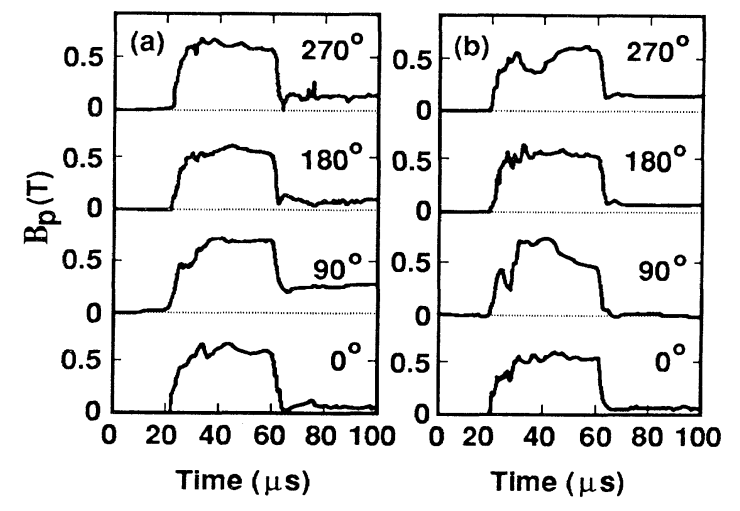

FIG. 2. (a) CT formation within the precompressor is usually stable and symmetric, as shown by the magnetic-field magnitude vs time at four azimuthal positions on the outer electrodes at $z=0.12 \mathrm{~m}$. (b) The early portion of CT formation is occasionally asymmetric but becomes symmetric within $30 \mu \mathrm{s}$. These data use hydrogen plasmas with the $10-\mu \mathrm{s}$-rise-time gun bank.

strate that the precompressor cone meets the goal of providing stability against tilt modes. ${ }^{18}$

The time to expel the CT from the precompressor, after the accelerator bank is fired, varies from about 10 $\mu \mathrm{s}$ at higher accelerator voltages to 20-40 $\mu$ s at lower voltages. In studying the low-voltage cases, we find that the magnetic field of the CT, decaying linearly to zero in about $60 \mu \mathrm{s}$, is decreasing faster than the crowbarred accelerator field, and that the CT is expelled from the precompressor only after the CT poloidal-magnetic-field magnitude has decayed to the order of the accelerating toroidal magnetic field. This is suggestive of a force balance between the acceleration field and the CT poloidal field pushing against the conical electrodes.

To evaluate the forces involved in compression, we compare the acceleration field with the CT field at an axial position of $0.43 \mathrm{~m}$, immediately after the precompressor cone, and show the results for 152 shots in Fig. 3. The accelerator field $\mathbf{B}_{\theta}$, following the CT and measured at the $8-\mathrm{cm}$ radius of the outer straight acceleration electrode, is plotted versus the peak value of the CT poloidal magnetic field $\mathbf{B}_{p}$. The $\boldsymbol{B}_{\theta}$ and $B_{p}$ values are each averaged over three probes at $0.43 \mathrm{~m}$. We find that the $B_{\theta}$ increases approximately linearly with $B_{p}$ over the large range of the data. The robustness of this result is shown by the close grouping of data taken under three substantially different sets of conditions: hydrogen plasmas with either a slowed fast-gun bank $(10 \mu \mathrm{s})$ or a slow-gun bank $(60 \mu \mathrm{s})$, and argon or neon plasmas with the slowgun bank. This result is reasonable because the CT fields exert a force against the precompressor cone proportional to $B_{p}^{2}$, pushing the CT toward the large end of the cone. This force opposes the force of the acceleration field proportional to $B_{\theta}^{2}$ that pushes the CT toward the small end of the cone, so in the quasistatic case, where 


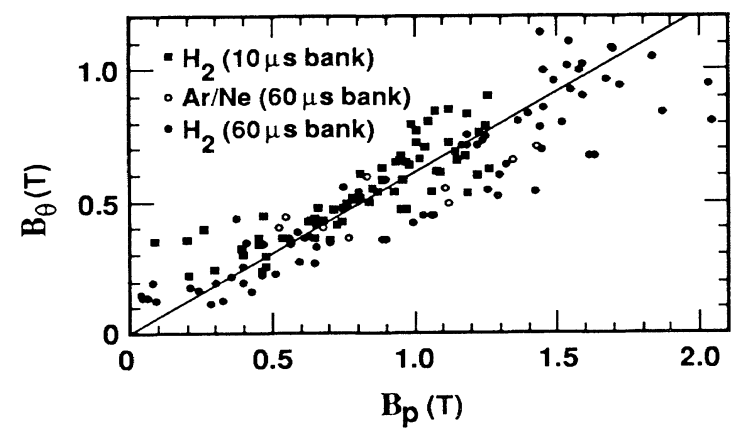

FIG. 3. The CT is in quasistatic pressure balance during compression in the conical electrodes. This is demonstrated by the linear increase of the $\mathbf{B}_{\theta}$ accelerator field with the $\mathbf{B}_{p}$ poloidal CT field, each the average of three probes separated by $90^{\circ}$ at $z=0.43 \mathrm{~m}$, the exit of the precompressor cone. Close agreement is observed among the data taken under three sets of conditions: hydrogen plasmas with either a slowed fast-gun bank $(10 \mu \mathrm{s})$ or a slow-gun bank $(60 \mu \mathrm{s})$, and argon or neon plasmas with the slow-gun bank. The line is the prediction of the 2D MHD code TRAC.

the forces balance, we expect $B_{\theta} \propto B_{p}$.

We compare these data with results obtained using the TRAC code, ${ }^{19}$ a $2 \mathrm{D}$ Lagrangian ideal MHD code that accurately models both time-dependent compression and acceleration. ${ }^{20}$ The line shown in Fig. 3 is from the code where the CT mass is chosen to scale proportionately with the CT magnetic energy $\left(M=100 \mu \mathrm{g}\right.$ at $U_{M}=3.1$ $\mathrm{kJ}, B_{p}=5 \mathrm{kG}$ before compression by an accelerator voltage of $80 \mathrm{kV}$ ). The agreement is seen to be quite satisfactory. Data can lie off the line because of errors in the probe measurements (the magnetic probe accuracy is estimated to be $\sim 20 \%$, including integration errors), or because of effects that are not included in the code, such as the Ohmic decay of the CT and thermal conduction.

The CT accelerates to velocities of $(2-6) \times 10^{7} \mathrm{~cm} / \mathrm{s}$ in the $1.7-\mathrm{m}$ straight accelerator used during these precompressor experiments. For example, as shown in Fig. 4 , the CT accelerates after emerging from the precompressor, as indicated by the increasing slope of the line through the magnetic-probe profiles, reaching a velocity $2.3 \times 10^{7} \mathrm{~cm} / \mathrm{s}$. Furthermore, the torus remains stable, as indicated by the nearly constant shape of the magnetic profiles throughout the acceleration period. Even these relatively low velocities, achieved with a short accelerator, are sufficient for tokamak fueling and current-drive experiments. ${ }^{4}$

A fast-opening switch ${ }^{8}$ appears possible, based on the observation that the $\mathbf{B}_{\theta}$ field behind the accelerated CT rises on a time scale $\sim 300 \mathrm{~ns}$ at the probes located on the outer electrode. The concept is that a load (e.g., a fast $z$ pinch) would be positioned at a gap in the inner (or outer) electrode. Current could be transferred to the load on the 300-ns time scale as the CT transits the gap. Since the time scale of inductive storage during the

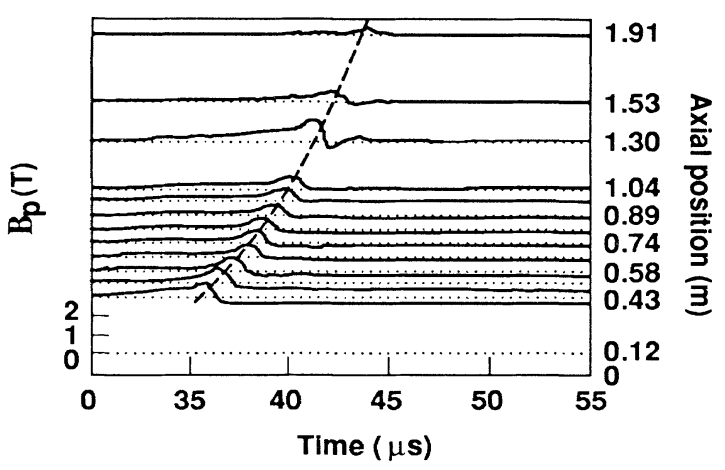

FIG. 4. The CT is accelerated and remains stable after emerging from the precompressor, as shown by the displaced but similar profiles from each $B_{p}$ probe for shot 6060 . The vertical offsets are proportional to the axial locations of each probe. The dashed line shows the CT trajectory. This shot uses hydrogen plasma with the $60-\mu \mathrm{s}$-rise-time gun bank.

compression phase can be $\sim 30 \mu \mathrm{s}$, a time compression factor of $\sim 100$ should be possible. The current rises by 300-600 kA in the $300 \mathrm{~ns}$, yielding a rate of rise of $(1-2) \times 10^{12} \mathrm{~A} / \mathrm{s}$. Efficient energy transfer will depend on maintaining a low plasma density embedded on the accelerating flux, since energy flow into the load cannot proceed faster than the Alfvén velocity $\left(\propto n^{-0.5}\right)$. Observations with a $\mathrm{HeNe}$ interferometer indicate typical line-average electron densities of a few percent of the peak CT densities $\left(n \sim 10^{16} \mathrm{~cm}^{-3}\right)$ in the following plasma. At accelerator energies greater than a few $M J$, opening times of $\leq 100 \mathrm{~ns}$ should be possible.

In conclusion, we have experimentally demonstrated a new technique for compression of CTs that both increases the suitability of accelerated CTs for several applications and reduces the level of technology required.

We gratefully acknowledge discussions with B. G. Logan, T. R. Jarboe, J. C. Fernández, and F. Wysocki, and the support of J. H. Nuckolls and J. F. Holzrichter. Capable engineering support was provided by D. B. Cummings and J. J. Delapena. This work was performed under the auspices of the U.S. Department of Energy at Lawrence Livermore National Laboratory under Contract No. W-7405-Eng-48.

${ }^{1}$ J. H. Hammer, C. W. Hartman, J. L. Eddleman, and H. S. McLean, Phys. Rev. Lett. 61, 2843 (1988).

${ }^{2}$ C. W. Hartman and J. H. Hammer, Phys. Rev. Lett. 48, 929 (1982).

${ }^{3}$ C. W. Hartman, J. L. Eddleman, J. H. Hammer, and D. L. Meeker, in Proceedings of the Fourth International Conference on Emerging Nuclear Energy Systems, Madrid, Spain, 1986, edited by G. Velarde and E. Minguez (World Scientific, Singapore, 1987), p. 158. 
${ }^{4}$ C. W. Hartman et al., in Proceedings of the Twelfth International Conference on Plasma Physics and Controlled Fusion Research, Nice, France, 1988 (IAEA, Vienna, 1989), Vol. III, p. 547.

${ }^{5}$ L. J. Perkins, S. K. Ho, and J. H. Hammer, Nucl. Fusion 28, 1365 (1988).

${ }^{6}$ S. K. Ho, L. J. Perkins, and J. H. Hammer, Fusion Technol. 17, 658 (1990).

${ }^{7}$ M. R. Brown and P. M. Bellan, Phys. Rev. Lett. 64, 2144 (1990).

${ }^{8}$ C. W. Hartman, J. Eddleman, and J. H. Hammer, in Proceedings of the IEEE International Conference on Plasma Science, Saskatoon, Canada, May 1986, IEEE Conference Record-Abstracts (IEEE, New York, 1986), p. 23, Paper No. $2 \mathrm{C} 4$.

${ }^{9} \mathrm{M}$. Yamada et al., in Proceedings of the Twelfth International Conference on Plasma Physics and Controlled Fusion Research (Ref. 4), Vol. II, p. 539.

${ }^{10}$ T. R. Jarboe et al., Phys. Fluids B 2, 1342 (1990).
${ }^{11}$ H. Alfvén, L. Lindberg, and P. Mitlid, J. Nucl. Energy 1, 116 (1960).

${ }^{12}$ W. C. Turner et al., in Proceedings of the Third Symposium on Physics and Technology of Compact Toroids (Los Alamos Scientific Laboratory Report No. LA-8700-C), p. 113.

${ }^{13}$ T. R. Jarboe et al., Phys. Rev. Lett. 45, 1264 (1980).

${ }^{14}$ C. W. Barnes et al., Phys. Fluids 29, 3415 (1986).

${ }^{15}$ J. B. Taylor, Phys. Rev. Lett. 33, 1139 (1974).

${ }^{16}$ F. J. Wysocki et al., in Proceedings of the Ninth U.S. Compact Toroid Symposium, Bellevue, Washington, March 1989 (Spectra Technology, Bellevue, WA, 1989), p. 29.

${ }^{17}$ T. R. Jarboe et al., Phys. Rev. Lett. 51, 39 (1983).

${ }^{18}$ M. N. Rosenbluth and M. N. Bussac, Nucl. Fusion 19, 489 (1979).

${ }^{19}$ J. L. Eddleman et al., Bull. Am. Phys. Soc. 34, 2051 (1989).

${ }^{20} \mathrm{~J}$. H. Hammer, in Proceedings of the Thirty-Second Annual Meeting of the Division of Plasma Physics, Cincinnati, November 1990 [Phys. Fluids B (to be published)]. 